\title{
Ultrafast decay and hydration dynamics of DNA bases and mimics
}

\author{
Samir Kumar Pal, Jorge Peon, Ahmed H. Zewail * \\ Laboratory for Molecular Sciences, Arthur Amos Noyes Laboratory of Chemical Physics, California Institute of Technology, \\ Pasadena, CA 91125, USA
}

Received 17 June 2002

\begin{abstract}
The ultrafast decay of the excited-state of DNA bases, reported here and elsewhere [Peon \& Zewail, Chem. Phys. Lett. 348 (2001) 255], makes very difficult the probing of the full hydration dynamics in DNA. Here we present studies of the natural DNA base mimic, 2-aminopurine (2AP), whose excited-state decay is much longer, in water and, for comparison, in bulk ethanol. Guided by these solvation results, which directly reflect the role of orientational and translational diffusion, we reexamine the ultrafast decay rates for the natural nucleotides and nucleosides. The results are important for the mechanism of energy disposal in DNA. (c) 2002 Elsevier Science B.V. All rights reserved.
\end{abstract}

\section{Introduction}

The dynamics of water in the close vicinity of a biomolecule is crucial to biological function [1] and plays a significant role in the molecular recognition of protein-ligand, protein-protein, protein-DNA and DNA-ligand systems. From this laboratory, recent emphasis in this area has centered around femtosecond dynamics of hydration at protein surfaces [2,3]. A key feature of these studies is the use of an intrinsic probe to obtain dynamical information without any disruption of the native structure of the macromolecule and with femtosecond resolution. For two different proteins, we have used for the interrogation a single

\footnotetext{
${ }^{*}$ Corresponding author. Fax: +1-626-792-8456.

E-mail address: zewail@caltech.edu (A.H. Zewail).
}

tryptophan molecule at the surface and in contact with water. In a recent theoretical study [4] the correlation between this solvation dynamics and the residence times of water in the hydration layer was shown to depend on the dynamical exchange with the bulk.

Studies of local reorganization in modified DNA strands have been reported recently $[5,6]$. By measuring time-resolved Stokes shift of an extrinsically inserted coumarin dye molecule to a deoxyribose of a DNA strand, these studies indicate a complex relaxation behavior on time scales ranging from $40 \mathrm{ps}$ to $40 \mathrm{~ns}$; the complementary strand has an abasic site analog opposite to the probe coumarin. The coumarin molecule was used in the above study in order to obtain information on the local environment in DNA. However, the lack of structural information on the coumarinDNA complex, from X-ray or NMR study, keeps 
unknown the extent of perturbation on the native local structure of DNA. On the other hand, the naturally occurring bases are not appropriate candidates to interrogate the full dynamics of hydration because of their ultrashort lifetimes in the excited states, as reported here and elsewhere using the direct fluorescence up-conversion method [7-9].

The analogue of adenine, 2-aminopurine (2AP), is commonly used as fluorescent sensor base and in this laboratory we have used it as a probe for studies of electron transfer in DNA duplexes and with nucleotide [10,11]. 2AP is structurally similar to the natural base adenine (A) (see Scheme 1) with almost the same properties of base pairing with thymine [12] and even cytosine [13]. NMR studies have recently confirmed that the replacement of $\mathrm{A}$ by $2 \mathrm{AP}$ does not perturb in a significant way the local structure of DNA [12-14]. Additionally there is evidence of conservation of biological function with this replacement $[15,16]$ as with other base mimics such as 7 -azaindole with a thymine analogue [17].

In this Letter, we report on the use of $2 \mathrm{AP}$ as a probe to interrogate the dynamics of hydration with femtosecond ( $\mathrm{fs}$ ) resolution in bulk water, and, for comparison, solvation in ethanol. The observed characteristic time constants in the constructed hydration correlation function of bulk water is similar to that obtained from other upconversion experiments using the tryptophan probe $[2,3]$. Hence, 2AP provides a unique opportunity for probing the total hydration and structural dynamics in DNA. Moreover, the time

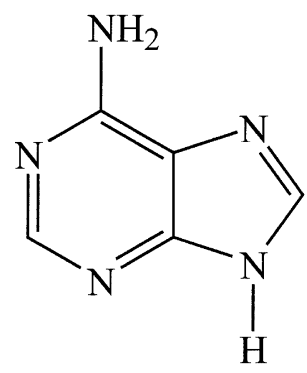

Adenine (A)

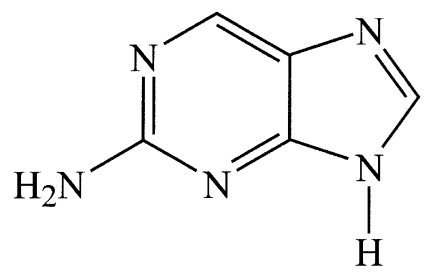

2-Aminopurine (2-AP)
Scheme 1. Molecular structures of the natural DNA base, adenine (A), and its fluorescent analog 2AP. scale of hydration is directly relevant in the studies of DNA nucleotides and nucleosides and here we consider such influence from analysis of decays for adenosine and thymidine.

\section{Experimental}

All experimental measurements were carried out by using femtosecond-resolved fluorescence up-conversion technique. The experimental setup is described elsewhere [2,3]. The femtosecond excitation pulse $(\sim 200 \mathrm{~nJ})$ from the tunable laser system was used at $297 \mathrm{~nm}$. The probe pulse was set at $800 \mathrm{~nm}$. The fluorescence from the sample was collected by a pair of parabolic focus mirrors and sent into a nonlinear crystal to mix with the probe pulse. The up-converted signal in the deep UV range (230-290 nm) was detected by a photomultiplier after dispersion through a double grating monochromator. For all transients the polarization axis of the pump beam was adjusted to be at $54.7^{\circ}$ (magic angle) with respect to the acceptance axis of the nonlinear crystal and the polarization axis of probe beam.

2AP was purchased from Sigma (99\% pure) and used without further purification. $100 \%$ ethanol (best grade) was acquired from Aaper alcohol. The aqueous solution of 2AP was prepared in water from a Nanopure purification system. The concentration of 2AP in aqueous and ethanol solutions were maintained to be 10 and $5 \mathrm{mM}$, respectively. Studies of nucleosides follow the same procedure reported in reference [7].

\section{Results and discussion}

\subsection{Hydration of the base $2 A P$}

As shown in Fig. 1 (top), 2AP in bulk water has an absorption maximum at $304 \mathrm{~nm}$. The position of excitation is shown by an arrow. In the emission spectra, 2AP in bulk water and ethanol show fluorescence maxima at 370 and $364 \mathrm{~nm}$, respectively, for $297 \mathrm{~nm}$ excitation. For comparison, the fluorescence spectrum of $2 \mathrm{AP}$ in the nonpolar solvent dioxane is also shown (maximum $355 \mathrm{~nm}$ 


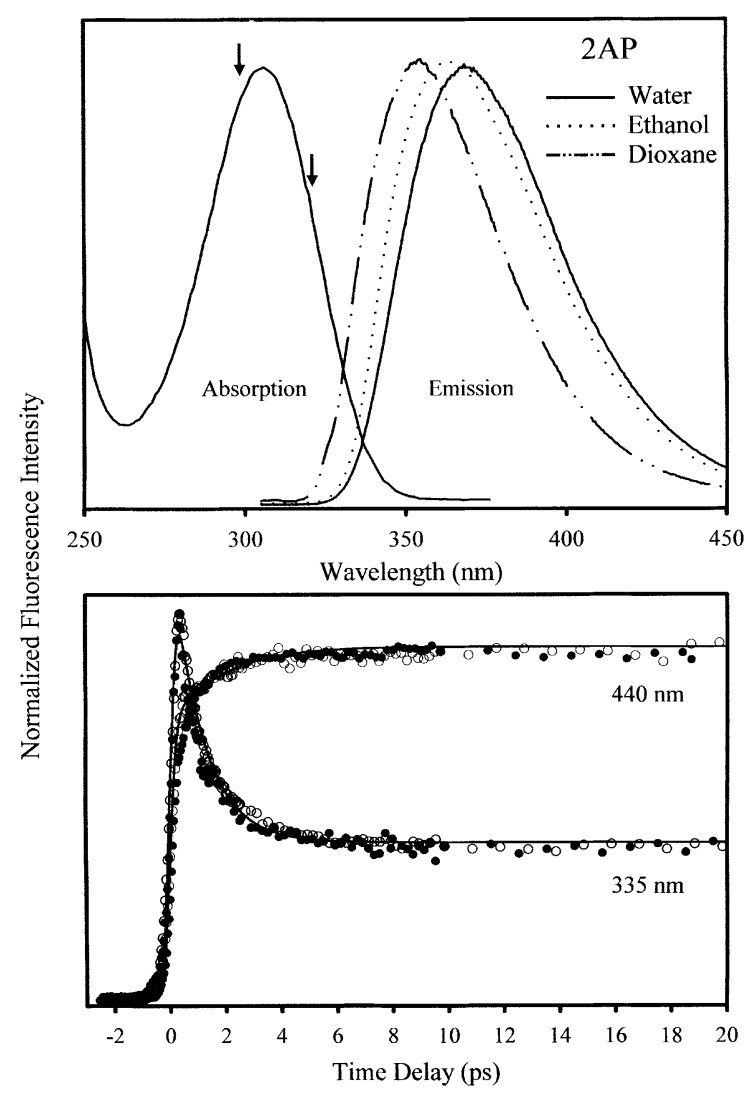

Fig. 1. The steady-state fluorescence spectra of the probe 2AP in different solvent environments (top). The absorption spectrum for 2AP in water is shown and the arrows mark the excitation wavelengths (297 and $320 \mathrm{~nm}$ ). The femtosecondresolved transients of $2 \mathrm{AP}$ in water are shown at two characteristic wavelengths, $335 \mathrm{~nm}$ (blue end) and $440 \mathrm{~nm}$ (red end) with excitation at $297 \mathrm{~nm}$ (bottom). The transients with solid circles indicate the temporal behavior with excitation at $320 \mathrm{~nm}$.

for excitation at $297 \mathrm{~nm}$ ). From the steady-state spectra, the solvatochromic shift of the fluorescence towards the longer wavelengths with the increase in solvent polarity is evident. Fig. 1 (bottom) displays the typical transients observed in the blue and red end of the spectrum in water, and shows the robustness of the behavior for two different excitation wavelengths (297 and $320 \mathrm{~nm}$ ).

The transients observed at different wavelengths, from the blue to the red side of the fluorescence spectrum of $2 \mathrm{AP}$ in water, are shown in Fig. 2. The emission transients detected in the blue region of the fluorescence spectrum are charac-

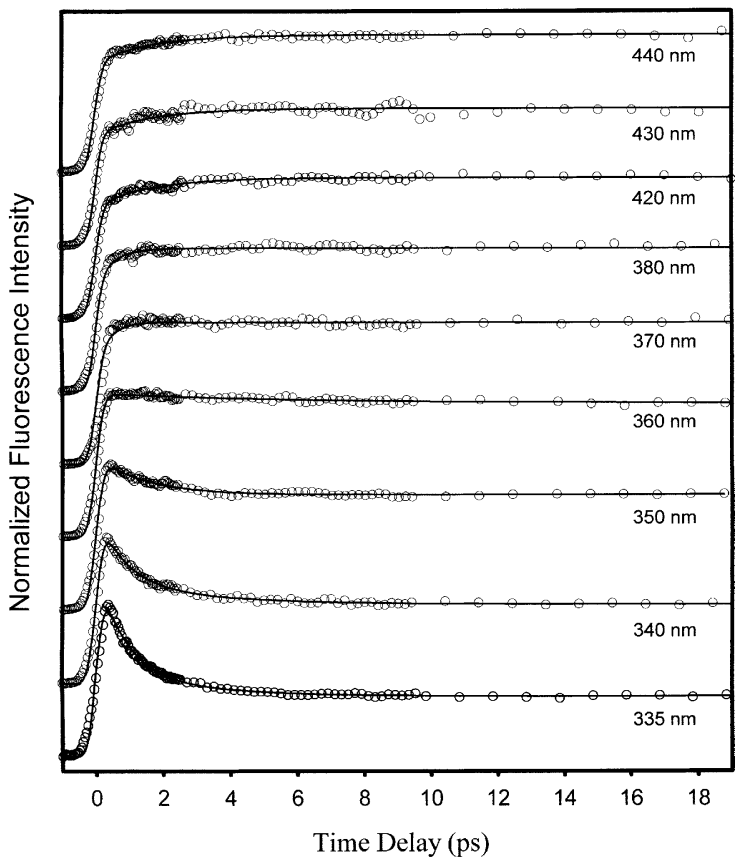

Fig. 2. Femtosecond-resolved transients of 2AP, spanning the different wavelengths of emission in pure water. The transients are normalized for comparison.

terized by an instant rise (cross-correlation) and typically a picosecond decay component. When detection is made in the red region, the decay part slows down until eventually an initial rise on a picosecond time scale is observed. The nanosecond decay was not measured here but was fixed at 11.8 $\mathrm{ns}$, which is the lifetime of $2 \mathrm{AP}$ in the relaxed equilibrium state [18]. These overall features are well recognized as being characteristic of solvation dynamics.

To construct the dynamical change of the spectra at various times, $t$, and the solvent response function, $C(t)$, we have adopted the procedure described in reference [19]. From the parameters of best fit to the emission transients and using the steady-state emission spectrum, time-resolved emission spectra (TRES) were constructed. The solvation dynamics is then described by the response function $C(t)$ which is defined as: $C(t)=[v(t)-v(\infty)] /[v(0)-v(\infty)]$, where $v(0)$, $v(t)$ and $v(\infty)$ denote the observed emission energies (in wavenumbers) at time $0, t$, and $\infty$, 


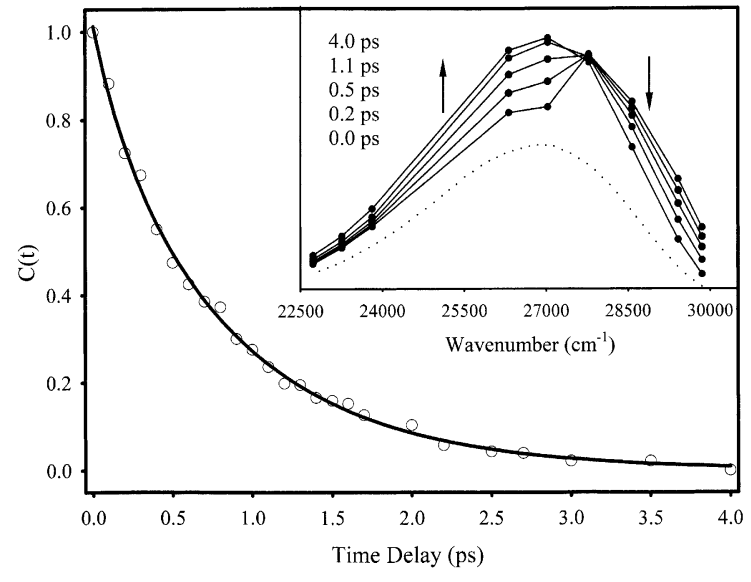

Fig. 3. The solvent response function constructed from femtosecond-resolved up-conversion measurements of 2AP in water. TRES (inset) at five delay times, from the initial $t=0$ and at longer times (see text). The arrows are displayed to indicate the decrease in intensity with time at the blue end and the corresponding rise at the red end, elucidating the spectral shift. The steady-state fluorescence spectrum of 2AP in water is also shown (dotted line) for illustration.

respectively. The time constants for solvation were determined from the temporal evolution of $C(t)$.

The inset of Fig. 3 depicts the TRES of 2AP in water. The steady-state fluorescence spectrum is shown in the figure (dotted line) for comparison with infinite time (4 ps in this case) spectrum. The time evolution of $C(t)$ is shown in Fig. 3, lower panel. The decay of the $C(t)$ was fitted to a biexponential function, giving two time constants $\sim 200 \mathrm{fs}(15 \%)$ and $870 \mathrm{fs}(85 \%)$; the $\sim 50$ fs or less component was not resolved. The net dynamical spectral shift is $610 \mathrm{~cm}^{-1}$ from $27140 \mathrm{~cm}^{-1}$ to $26530 \mathrm{~cm}^{-1}$ (4 ps). The temporal behavior of the $C(t)$ is very similar to that observed recently using the aminoacid tryptophan as solvation probe [2]. This is consistent with the fact that both probes interrogate the same dynamics of bulk water; both increase their dipole moments upon excitation for tryptophan it is $\sim 6 \mathrm{D}$, while for $2 \mathrm{AP}$ the increase is estimated to be $(1.6 \leqslant \Delta \mu \leqslant 2.9 \mathrm{D})$ [21].

\subsection{Solvation vs. vibrational relaxation}

The observed behavior of $C(t)$ on the subpicosecond time scale result from solvation pro- cesses; vibrational relaxation is relatively insignificant for several reasons. First, as shown in Fig. 1 (bottom), the behavior of the transients is the same for excitation at 297 or $320 \mathrm{~nm}$. The theoretically predicted lowest transition energy for 2AP chromophore $\left(S_{0} \rightarrow S_{1}\right), \pi-\pi^{*}$ in character, is estimated to be at $33550 \mathrm{~cm}^{-1}$ in the gas phase [18], and our excitation energy is close to such a value (see also the arrows in Fig. 1). Similar to tryptophan, which was excited near the zero vibrational energy state, the excess vibrational energy for 2AP is not significant. Second, the shape of TRES at early times is not significantly different from those obtained for later times, consistent with the lack of vibrational relaxation at our energies and time scale of $C(t)$. It should be mentioned that vibrational relaxation of adenosine in water [20] occurs on a few picoseconds, but for an excess energy of $\sim 34000$ $\mathrm{cm}^{-1}$. Finally, the observed relaxation dynamics in ethanol is found to be much slower than that in water, confirming the above conclusion regarding the evolution of $C(t)$.

In bulk ethanol, at the blue side $(335 \mathrm{~nm})$ the transient shows a decay of 14 ps $(50 \%)$ and a nanosecond component (again, the $11.8 \mathrm{~ns} ; 50 \%$ ), as shown in Fig. 4. At the red end $(440 \mathrm{~nm})$ of the spectrum, a rise with a time constant of $28 \mathrm{ps,}$ together with the nanosecond (11.8 ns) component, was observed. The temporal behavior in Fig. 4 is a clear indication of the slower solvation process. The reported [22] average solvation time for ethanol is $16 \mathrm{ps}$, which is only slightly longer than our observed decay (14 ps) at the blue end. The rise component at the red end ( $28 \mathrm{ps})$ is also close to the longest time constant of the reported solvation relaxation (29.6 ps). Another confirmation of the observed slow relaxation comes from dielectric relaxation measurements by Barthel et al. [23]. The study confirmed the existence of three dispersion regions with solvent longitudinal dielectric relaxation time constants of 1.3, 7.6 and 30 ps. The reported longest longitudinal relaxation time constant ( $30 \mathrm{ps}$ ) is in a close agreement with our observation of the rise component ( 28 ps) at the red end of the fluorescence spectrum of 2AP in ethanol; if $C(t)$ was constructed for ethanol, then we could resolve the other relaxation time(s). 


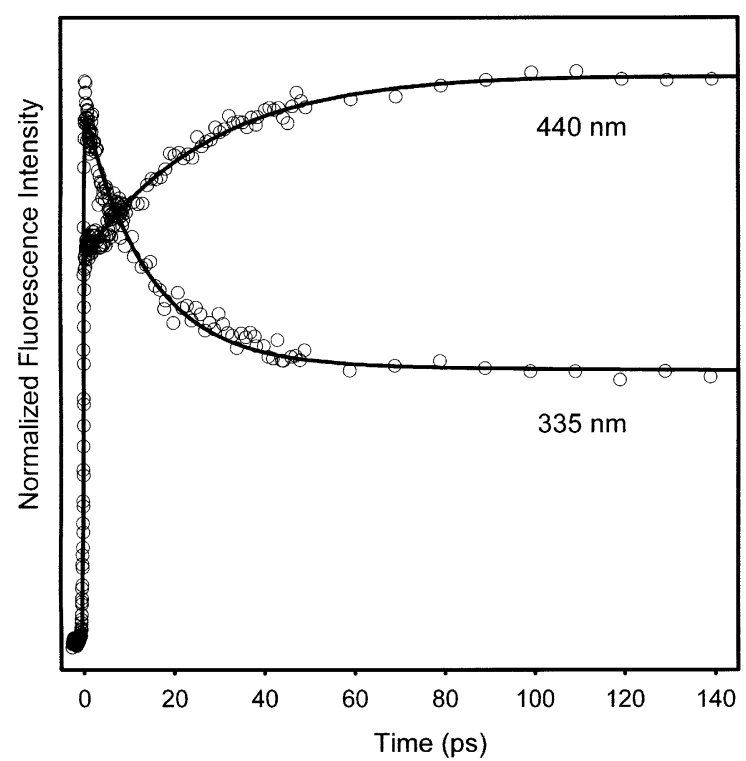

Fig. 4. Time-resolved fluorescence transients of $2 \mathrm{AP}$ in ethanol. Note the distinct difference from the transients in bulk water.

\subsection{The natural DNA bases}

As mentioned above, the native bases of DNA, in contrast to 2AP, have ultrashort excited-state decays, as reported by this laboratory, using fluorescence up-conversion [7], and by other groups, using transient absorption [20,24,25]. Our initial report of direct lifetime measurements of eight nucleotides and nucleosides support the conclusion that all these molecules undergo internal conversion to the ground state on the sub-picosecond time scale. In that study [7], we analyzed the data by considering a single exponential decay of the up-conversion signal.

In view of the above results of femtosecond hydration we refitted here the data to a biexponential function. In more recent study by [8,9], the up-conversion transients for adenine and thymine nucleosides/nucleotides were fitted to a biexponential function, the fastest being sub-100 to 200 $\mathrm{fs}$, and the other decay time ranged from $0.5 \mathrm{ps}$, for adenine nucleoside/nucleotide, to 0.69 and 1.1 ps, for thymine's nucleoside and nucleotide, respectively. These deconvolution of decays in both studies give agreement for the longer time constants [7-9]. Our data on the nucleosides/nucleo- tides can be fitted to the same biexponential function, but, as shown in Fig. 5, the difference is not significant enough for us draw major conclusions. However, if fitted to a biexponential form, then we obtained the shortest decay to be $\sim 200 \mathrm{fs}$. In our current analysis we consider a revised instrument response time of $450 \pm 100 \mathrm{fs}$ (somewhat different from the 360 fs value [7]).

With the response time obtained from the analysis of the instrument-limited rise-times in $2 \mathrm{AP}$ in ethanol and biphenyl-carboxilic acid in methanol as well as from the up-conversion of water's Raman scattering, we obtained the fits in Fig. 5: the up-conversion transients for adenosine

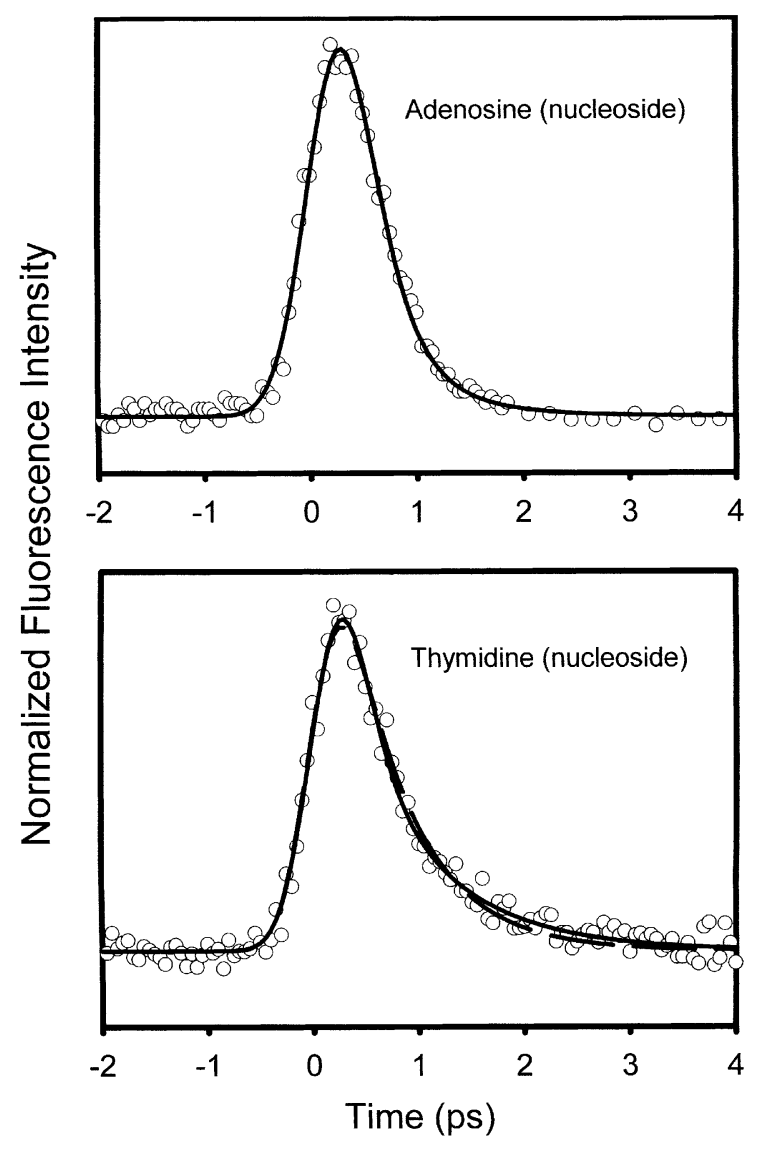

Fig. 5. Femtosecond-resolved transients of the nucleosides of the natural bases adenine (adenosine) (top) and thymine (thymidine) (bottom). Note the small difference between the biexponential (solid line) and single-exponential (dotted line) fits (see text). 
(top, $\lambda_{\text {fluo }}=320 \mathrm{~nm}$ ) and thymidine (bottom, $\lambda_{\text {fluo }}=330 \mathrm{~nm}$ ) for an excitation wavelength of 270 $\mathrm{nm}$. The solid lines correspond to the convoluted biexponential decays with $\tau_{1} \approx 0.2$ ps $(85 \%)$ and $\tau_{2}=0.5 \mathrm{ps}(15 \%)$ for adenosine and with $\tau_{1} \approx 0.2$ ps $(71 \%)$ and $\tau_{2}=0.8$ ps $(29 \%)$ for thymidine. For the nucleotide adenosine $5^{\prime}$-monophosphate (AMP), the same decay times were observed as for adenosine (within our signal-to-noise). For thymidine $5^{\prime}$-monophosphate (TMP) we obtained $\tau_{1} \approx 0.2 \mathrm{ps}$ $(62 \%)$ and $\tau_{2}=1.2$ ps $(38 \%)$. In Fig. 5 (bottom), we include the result of a single exponential fit for comparison.

For thymidine 5'-monophosphate (TMP) in water we have checked for the behavior of the transients with fluorescence wavelength. As shown in reference [7], all transients in the wavelength region of $310-350 \mathrm{~nm}$, covering the blue and red side, display decays to the base line, with no longlived component; at our signal-to-noise level the overall decay time is within $30 \%$. This conclusion is consistent with the study made in reference [8]. We therefore conclude that the lifetime is indeed 1 ps or less and that solvation may only contribute on the femtosecond time scale.

\section{Conclusion}

Hydration dynamics of 2AP in water was observed, with our time resolution, to be ultrafast, $\sim 200$ and $870 \mathrm{fs}$, but in ethanol solvation is much longer (16 ps), elucidating the potential for using $2 \mathrm{AP}$ as probe of hydration in DNA. We have conducted some preliminary experiments of $2 \mathrm{AP}$ in well-defined DNA duplexes [10] and the full study will be published elsewhere. Because excitedstate lifetimes of naturally occurring DNA bases are ultrafast they cannot be used to probe the full range of hydration dynamics, but the role of hydration, especially in the early domain of femtoseconds, cannot be completely ignored. This is particularly relevant when one is addressing the mechanism of energy disposal by, e.g., conical intersections, as suggested by Sobolewski and Domcke and their colleagues [26,27]. Independent of the initial $\sim 100-200 \mathrm{fs}$ relaxation, all studied nucleosides and nucleotides (reference [7] and here) dispose their energy in water in less than 1 ps. The biological significance is clear.

\section{Acknowledgements}

This work was supported by the National Science Foundation. We thank Prof. Biman Bagchi for stimulating discussion on the theoretical aspects of solvation.

\section{References}

[1] R. Pethig, Annu. Rev. Chem. 43 (1992) 177.

[2] S.K. Pal, J. Peon, A.H. Zewail, Proc. Natl. Acad. Sci. USA 99 (2002) 1763.

[3] J. Peon, S.K. Pal, A.H. Zewail, Proc. Natl. Acad. Sci. USA (in press).

[4] S.K. Pal, J. Peon, B. Bagchi, A.H. Zewail, J. Phys. Chem. (in press).

[5] E.B. Brauns, M.L. Maradas, R.C. Coleman, C.J. Murphy, M.A. Berg, J. Am. Chem. Soc. 121 (1999) 11644.

[6] E.B. Brauns, M.L. Maradas, R.C. Coleman, C.J. Murphy, M.A. Berg, Phys. Rev. Lett. 88 (2002) 158101.

[7] J. Peon, A.H. Zewail, Chem. Phys. Lett. 348 (2001) 255.

[8] T. Gustavsson, A. Sharonov, D. Onidas, D. Markovitsi, Chem. Phys. Lett. 351 (2002) 195.

[9] T. Gustavsson, A. Sharonov, D. Onidas, D. Markovitsi, Chem. Phys. Lett. 356 (2002) 49.

[10] C. Wan, T. Fiebig, O. Schiemann, J.K. Barton, A.H. Zewail, Natl. Acad. Sci. USA 97 (2000) 14052.

[11] T. Fiebig, C. Wan, A.H. Zewail, Chem. Phys. Chem. (in press).

[12] T.M. Nordlund, S. Andersson, L. Nilsson, R. Rigler, A. Gräslund, L.W. McLaughlin, Biochemisty 28 (1989) 9095.

[13] L.C. Sowers, G.V. Fazakerley, R. Eritja, B.E. Kaplan, M.F. Goodman, Proc. Natl. Acad. Sci. USA 83 (1986) 5434.

[14] L.W. McLaughlin, T. Feong, F. Benseler, N. Piel, Nucleic Acids Res. 16 (1988) 5631.

[15] B. Holz, S. Klimasauskas, S. Serva, E. Weinhold, Nucleic Acids Res. 26 (1998) 1076.

[16] B.W. Allan, N.O. Reich, J.M. Beechem, Biochemistry 38 (1999) 5308.

[17] Y. Wu, A.K. Ogawa, M. Berger, D.L. McMinn, P.G. Schultz, F.E. Romesberg, J. Am. Chem. Soc. 122 (2000) 7621.

[18] A. Holmén, B. Nordén, B. Albinsson, J. Am. Chem. Soc. 119 (1997) 3114.

[19] M. Maroncelli, G.R. Fleming, J. Chem. Phys. 86 (1987) 6221.

[20] J.-M.L. Pecourt, J. Peon, B. Kohler, J. Am. Chem. Soc. 123 (2001) 10370.

[21] E.L. Rachofsky, J.B.A. Ross, M. Krauss, R. Osman, Acta Phys. Polonica A 94 (1998) 735. 
[22] M.L. Horng, J.A. Gardecki, A. Papazyan, M. Maroncelli, J. Chem. Phys. 99 (1995) 17311.

[23] J. Barthel, K. Bachhuber, R. Buchner, H. Hetzenauer, Chem. Phys. Lett. 165 (1990) 369.

[24] D.N. Nikogosyan, D. Angelov, B. Soep, L. Lindqvist, Chem. Phys. Lett. 252 (1996) 322.
[25] A. Reuther, H. Iglev, R. Laenen, A. Laubereau, Chem. Phys. Lett. 325 (2000) 360.

[26] A.L. Sobolewski, W. Domcke, C. Dedonder-Lardeux, C. Jouvet, Phys. Chem. Chem. Phys. 4 (2002) 1093.

[27] A.L. Sobolewski, W. Domcke, Europhys. J. (in press). 